\title{
Exploration du rôle potentiel des pharmacies privées dans l'offre de services de planification familiale au Sénégal: Revue documentaire sur les aspects juridiques
}

\author{
Fatou Mbow \\ Population Council \\ Ndeye Dome Fall \\ Nafissatou Diop \\ Population Council \\ Sara Chace Dwyer \\ Population Council
}

Follow this and additional works at: https://knowledgecommons.popcouncil.org/departments_sbsr-rh How does access to this work benefit you? Let us know!

\footnotetext{
Recommended Citation

Mbow Fatou, Ndeye Dome Fall, Nafissatou Diop, and Sara Chace Dwyer. 2018. "Exploration du rôle potentiel des pharmacies privées dans l'offre de services de planification familiale au Sénégal: Revue documentaire sur les aspects juridiques," Technical brief. Dakar: Population Council, The Evidence Project.
} 


\section{Exploration du rôle potentiel des pharmacies privées dans l'offre de services de planification familiale au Sénégal :}

Revue documentaire sur les aspects juridiques

Au Sénégal, le Plan d'Action National de Planification Familiale (PANPF) pour la période 2012-2015 et le Cadre Stratégique National pour la Planification Familiale (CSNPF) 2016-2020 qui s'en est suivi ont adopté une approche multisectorielle, faisant du secteur privé et du niveau communautaire, des partenaires clés du secteur public dans sa mise en œuvre pour élargir l'accès des services de Planification Familiale (PF) et atteindre l'objectif de 45\% de Taux de Prévalence Contraceptive moderne (TPCm) en 2020. Cependant, le secteur privé à but lucratif, en particulier les pharmacies privées, ne participe pas activement à l'offre de services de PF en raison de l'existence de barrières, notamment juridiques, qui limitent leur rôle au conseil et à la vente de produits contraceptifs. Cette recherche a été réalisée grâce au soutien financier de l'Agence des Etats Unis pour le Développement International (USAID) à travers le Projet Evidence, et la Fondation William et Flora Hewlett à travers un financement au Population Council.

\section{TECHNICAL BRIEF}

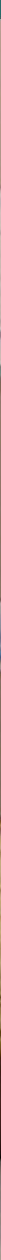




\section{Introduction}

En 2012, la Direction de la Santé de la Reproduction et de la Survie de l'Enfant (DSRSE) du Ministère de la Santé et de l'Action Sociale (MSAS) a adopté un ambitieux PANPF pour la période 2012-2015. Celui-ci visait à accroitre le TPCm de 12\% en 2012 à $27 \%$ en 2015, et à 45\% en 2020 en augmentant l'accès des populations aux services de PF. Entre 2012 et 2014, le TPCm a fait des bonds annuels de quatre (4) points de pourcentage, pour atteindre 20\% en 2014 (EDS-C, 2013 ; EDS-C, 2014). Toutefois, le rythme de progression a diminué en 2015 avec un TPCm de 21\% (EDS-C 2015). De plus, les besoins non satisfaits en PF estimés à 25\% en 2015 (EDS-C 2015) parmi les femmes en union restent élevés. À la suite du PANPF 20122015, un nouveau CSNPF a été adopté pour la période 2016-2020, avec le même objectif de 45\% de TPCm en 2020.

C'est dans ce cadre, qu'une étude exploratoire sur le rôle potentiel des pharmacies privées dans l'offre de services de PF au Sénégal a été lancée en 2015 par le Population Council, dans le cadre du Projet Evidence, en collaboration avec la DSRSE, le Bureau santé de I'USAID au Sénégal, l'Agence pour le Développement du Marketing Social, I'Unité de Coordination du Partenariat de Ouagadougou et I'Institut de Population, Développement et Santé de la Reproduction. L'étude a été financée par I'USAID et la Fondation William et Flora Hewlett. L'une des composantes de l'étude était une revue documentaire sur les aspects juridiques de l'offre de services de PF par les pharmacies privées qui fait l'objet de ce résumé (les trois autres composantes ainsi que la synthèse de l'étude sont disponibles sur le site du Projet Evidence). Cette revue a analysé les lois et règlements en vigueur afin d'identifier les arguments en faveur et à l'encontre de l'offre des services de PF par les pharmacies privées, et de faire des suggestions sur les révisions nécessaires pour permettre cette offre.

L'étude a produit des données probantes, en appui au plaidoyer en faveur d'une plus grande implication des pharmacies privées dans l'offre de services de PF, conformément à l'approche multisectorielle prônée par le Gouvernement du Sénégal afin de mieux répondre aux besoins de PF et de Santé de la Reproduction (SR) des personnes et des couples au Sénégal.

\section{Méthodologie}

L'approche méthodologique a consisté en un recensement des textes législatifs et réglementaires régissant le secteur privé de manière générale au Sénégal, et plus particulièrement l'exercice de la profession de pharmaciens privés, ainsi que la réglementation applicable aux substances vénéneuses. Au total, 11 documents ont été identifiés, parmi lesquels deux (2) textes de lois, trois (3) décrets, deux (2) accords internationaux, deux (2) documents de politique nationale, une circulaire et un rapport de recherche. Le contenu de ces documents a été analysé par extraction et classification des informations correspondants aux thèmes retenus.

\section{Résultats}

Le Graphique 1 ci-dessous montre les services de PF pouvant être offerts par les différentes catégories d'agents de santé sur la base des documents de politiques, normes et protocoles disponibles à la date de rédaction du présent document. II apparait que les pharmacies privées jouent le plus petit rôle puisqu'il se limite à l'offre de conseils, la vente de produits de PF et la référence des client(es) en cas de besoin.

\section{Les obstacles juridiques à l'offre de services de PF par les pharmacies privées}

L'exercice de la pharmacie d'officine est régi par un texte de loi du 15 avril 1954 portant application du Code de Santé Publique (CSP) français. Ce code, précise en son Article 569 que " l'exploitation d'une officine est incompatible avec l'exercice d'une autre profession, notamment celle de médecin, vétérinaire, sage-femme, dentiste, même si l'intéressé est pourvu des diplômes correspondants ". Ceci suppose que le pharmacien d'officine ne peut s'adonner à des actes médicaux (par exemple faire une injection) 


\section{GRAPHIQUE 1}

TÂCHES ET RESPONSABILITÉS DU PERSONNEL DE SANTÉ EN MATIÈRE DE PF SUIVANT LES POLITIQUES, NORMES ET PROTOCOLES EN SANTÉ DE LA REPRODUCTION

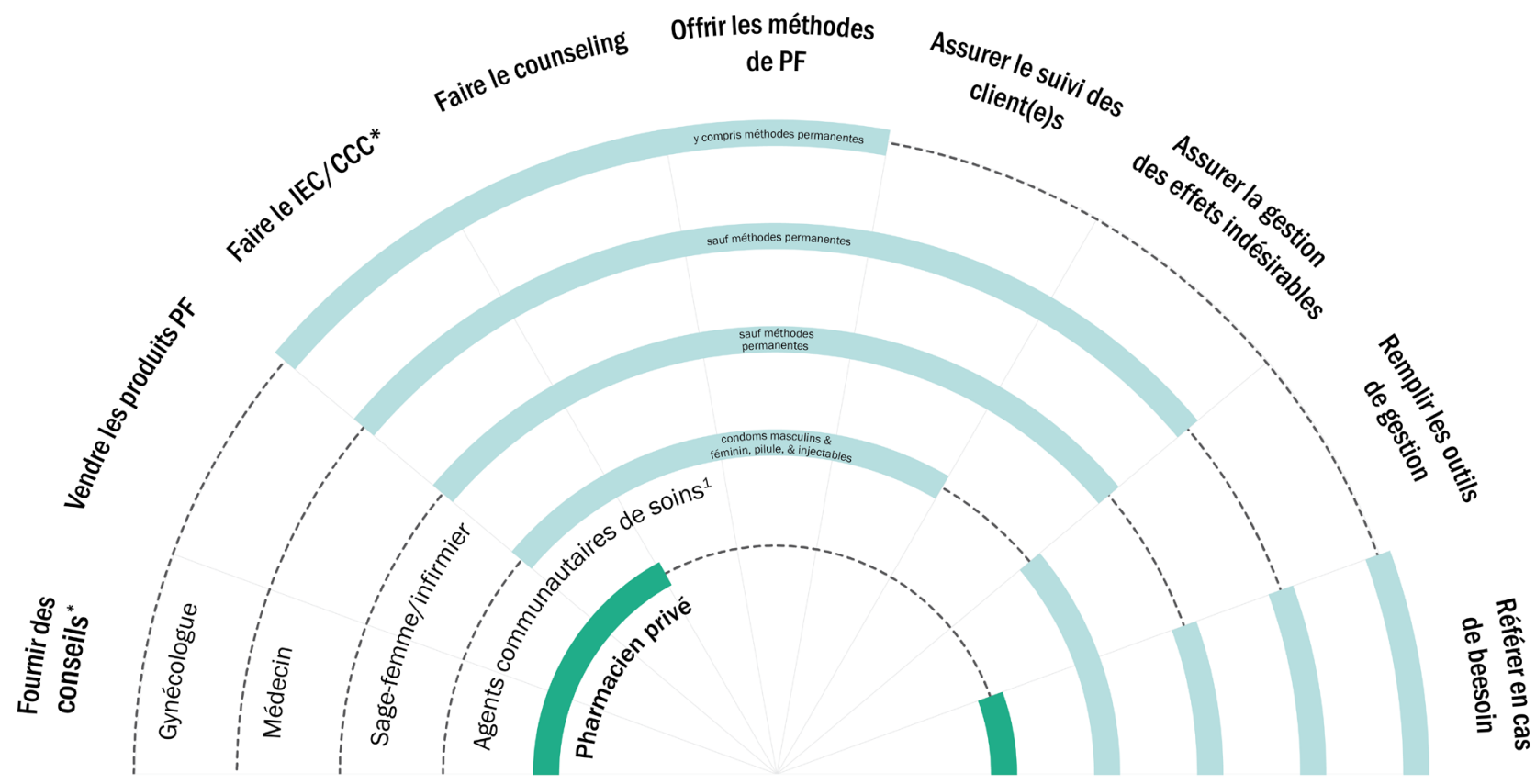

*Information, éducation et communication/Communication pour le changement de comportement

qui sont dévolus à ces professionnels de santé. Cette disposition est corroborée par l'Article 72 du Décret de 1981 portant Code de déontologie des pharmaciens qui indique que : " ... le pharmacien d'officine doit veiller à ce que des consultations ou actes médicaux ne soient jamais pratiqués dans l'officine par qui que ce soit ".

Ainsi, malgré le grand rôle que ces acteurs jouent en tant que premier recours au système de santé, les actes autorisés aux pharmaciens se limitent en grande partie à la délivrance/vente de produits et à l'offre de conseils. Ils ne peuvent pas fournir des services de PF tels que le counseling ou la prescription de produits contraceptifs. Ils sont tenus de référer au besoin les patients dans les structures de santé. Ceci renvoie à la conclusion de certains auteurs qui soulignent l'interdiction qu'il puisse y avoir en même temps dans un établissement privé des prestations de services de counseling, de prescription et de vente de produits contraceptifs (Wickstrom, Diagne, et Smith, 2006). Par exemple, puisque la pharmacie vend des produits contraceptifs, il lui est interdit de faire le counseling ou de prescrire ces produits. De même, une clinique privée a interdiction de stocker et de vendre des produits contraceptifs.

Par ailleurs, la plupart des produits vendus en officine exigent la présentation d'une ordonnance. C'est le cas de certains produits contraceptifs qui, comme tout produit inscrit sur les listes I et II de la nomenclature des médicaments, sont soumis à la réglementation des substances vénéneuses. ${ }^{2}$ En effet, la délivrance de ces produits ne peut se faire que sur présentation d'une ordonnance. ${ }^{3}$ Ainsi, pour la prescription d'un contraceptif listé, les client(e)s doivent préalablement consulter un médecin, un infirmier ou une sage-femme exerçant dans une structure sanitaire (publique ou privée)

\footnotetext{
1 Nouvelle appellation des agents communautaires de soins.

2 Médicaments inscrits sur la liste I : spécialités présentant les risques les plus élevés. Médicaments inscrits sur la liste II : substances vénéneuses considérées comme moins dangereuses.

3 Actuellement au Sénégal, concernant les contraceptifs oraux, seule la contraception d'urgence peut être délivrée aux populations sans ordonnance par les officines de pharmacie privées compte tenu de la spécificité du produit.
} 
avant d'amener l'ordonnance à la pharmacie privée pour délivrance.

En outre, la Loi du $\mathbf{5}$ août $\mathbf{2 0 0 5}$ relative à la SR énonce en son Article 8 que : "Le personnel intervenant dans le domaine de la santé de la reproduction est tenu de se soumettre aux normes de compétence, aux protocoles de services et aux règles de déontologie afférentes à sa profession ou à son activité ". C'est dire que les pharmaciens ont l'obligation de s'en tenir aux règles édictées par la réglementation pharmaceutique et également leur code de déontologie. Ils ne peuvent donc pas offrir des services de PF tel que le counseling, la prescription ou l'administration des méthodes.

\section{Les opportunités en matière d'offre de services de PF par les pharmacies privées}

Malgré l'existence de barrières juridiques à l'implication des pharmacies privées dans l'offre de services de PF, des opportunités existent au regard de certaines dispositions. Par exemple, les principaux accords internationaux signés par le Sénégal reconnaissent le droit à la PF. II s'agit notamment de la Convention sur l'élimination de toutes les formes de discrimination à l'égard des femmes (CEDEF 1979/Partie III, Articles 10, 12 et 14) qui précise que les États signataires sont tenus de fournir aux femmes avant la grossesse, pendant la grossesse, au cours de l'accouchement et après l'accouchement, des services appropriés. Par ailleurs, le Protocole à la Charte africaine des droits de l'Homme et des peuples relatif aux droits des femmes en Afrique du 11 juillet 2003 (plus connu sous le nom de Protocole de Maputo) stipule en son Article 14 que les États doivent veiller au respect et à la promotion des droits de la femme à la santé, y compris la santé sexuelle et reproductive. Ces droits comprennent le libre choix des méthodes contraceptives. Les États doivent également prendre toutes les mesures appropriées pour " assurer l'accès des femmes aux services de santé adéquats, à des coûts abordables et à des distances raisonnables, y compris les programmes d'information, d'éducation et de communication pour les femmes, en particulier celles vivant en milieu rural. "En outre, la loi de 2005 relative à la SR garantit également aux populations le droit d'accéder aux services, y compris ceux de la PF, même si elle ne se prononce pas sur le personnel compétent pour offrir ces services.

Le Sénégal a donc l'obligation de respecter ses engagements internationaux et nationaux et a la responsabilité de veiller au respect de ces droits pour ses citoyens. L'élargissement du rôle des pharmacies privées dans l'offre des services de PF est une solution offerte au Sénégal pour respecter ses obligations et augmenter l'accès des populations à la $\mathrm{PF}$.

Le décret portant Code de déontologie des pharmaciens, quant à lui impose aux pharmaciens l'obligation de " respect de la vie et de la personne humaine " ce qui " constitue en toute circonstance, un devoir primordial " (Article 2). Respecter la vie et la personne humaine, signifie entre autres, respecter tous ses droits, notamment, sexuels et reproductifs. A cet égard, le pharmacien doit pouvoir contribuer au respect du droit de chaque personne de se protéger contre les grossesses non désirées, d'accéder aux services de PF et de bénéficier de la promotion des services de SR. Aux termes de l'Article 5 du même Code, "le pharmacien est au service du public. II doit faire preuve du même dévouement envers tous les malades ". À cet effet, toute femme désireuse de contraception doit être traitée de manière égale, quel que soit son profil, et ses besoins doivent être respectés comme ceux de tout autre client. L'Article 7 du même Code se montre encore plus pertinent en précisant que "Les pharmaciens sont tenus de prêter leur concours aux services de médecine sociale et collaborer à l'œuvre des pouvoirs publics tendant à la protection et à la préservation de la santé publique ". Ainsi, en tant qu'acteurs de santé publique, les pharmaciens devraient pouvoir, au-delà du conseil et de la délivrance/vente de produits contraceptifs, apporter une plus grande contribution à l'offre de services de PF.

De plus, suite à l'autorisation accordée aux agents de santé communautaire en 2012 de faire l'offre 
initiale de pilules à l'issue d'une expérience pilote, l'offre initiale de contraceptifs injectables sous forme intramusculaire (Depo Provera) et souscutanée (Sayana Press) par ces mêmes agents dans tous les districts sanitaires a été règlementée à travers une circulaire de 2014 , et cela conformément au cadre de passage à l'échelle des expériences communautaires réussies. De ce fait, le rôle des pharmaciens, qui ont des qualifications supérieures aux agents communautaires de soins, devrait pouvoir être renforcé dans ce domaine.

\section{Discussion}

Au Sénégal, l'implication des pharmacies privées dans I'offre de services de PF se heurte encore à de nombreux obstacles législatifs et règlementaires. Ces obstacles sont essentiellement liés aux règles de fonctionnement des établissements du secteur privé, dont font partie les pharmacies, et aux devoirs des pharmaciens, tels que conçus par le code qui régit leur profession et définit les règles qu'ils doivent observer dans leurs relations avec le public. Ces obstacles sont également liés à la nature de la plupart des méthodes contraceptives, et par conséquent à la place qu'elles occupent dans la nomenclature des médicaments (par exemple la nécessité d'une ordonnance).

Des avancées importantes ont été notées dans le PANPF 2012-2015 avec la volonté de mobiliser l'ensemble des acteurs de la santé, notamment ceux du secteur privé, grâce à une approche multisectorielle pour l'atteinte des objectifs fixés. Cette ambition a été renouvelée avec le CSNPF de 2016-2020. Malgré les barrières juridiques qui empêchent actuellement les pharmacies privées de contribuer significativement à la hausse du TPCm, leur implication future n'est pas exclue. En effet, certaines dispositions législatives et réglementaires mettant en avant les droits et issues, pour beaucoup, des instruments internationaux et régionaux obligent le Sénégal à offrir un accès équitable aux informations et aux services de PF. Comme les pharmacies privées sont déjà nombreuses, vendent des produits de $\mathrm{PF}$, et sont proches des populations, une plus grande inclusion de ces dernières dans I'offre des services de PF pourra être une solution pour se conformer à ces instruments. II y a également des textes régissant la profession pharmaceutique qui offrent des opportunités pour leur meilleure implication dans l'offre de services de PF. Il devient donc urgent de faire du personnel des pharmacies privées, des acteurs à part entière dans l'offre de services de PF afin d'améliorer l'accès des populations aux méthodes contraceptives et atteindre les objectifs de développement durable.

\section{Recommandations}

Les recommandations suivantes ont été formulées :

- Insérer dans le Décret portant Code de déontologie des pharmaciens des dispositions plus explicites sur le rôle attendu d'eux en matière de PF.

- Proposer un décret portant sur l'exercice de la profession de pharmacien d'officine en incluant dans ses missions l'offre des services de PF.

- Proposer un décret d'application de la loi relative à la SR de 2005, fixant clairement les normes de compétence, les protocoles de services et les règles propres à chacune des catégories professionnelles.

\section{Conclusion}

Au Sénégal, les pharmacies privées, bien qu'étant les premiers points de contact de nombreuses personnes au système de santé, ne jouent qu'un rôle très limité dans l'offre de services de PF. Dans le contexte actuel, les pharmaciens ne jouent pas un grand rôle dans l'offre des services de PF, compte tenu du cadre juridique et règlementaire existant. Pourtant des opportunités d'augmenter leur contribution existent. En effet, l'analyse de certaines dispositions de pactes et conventions internationaux et régionaux, ainsi que certains textes au niveau national laisse penser que l'offre des services de PF par les pharmacies privées peut être envisagée. Cependant, le défi réside dans la levée, dans les meilleurs délais, des obstacles identifiés à travers une révision des lois et des dispositions règlementaires problématiques. 


\section{RÉFÉRENCES BIBLIOGRAPHIQUES}

Convention sur l'élimination de toutes les formes de discrimination à l'égard des femmes (CEDEF). 1979/Partie III.

Décret $n^{\circ}$ 81-039 du 2 février 1981 portant Code de déontologie des pharmaciens.

Décret $n^{\circ}$ 96-395 du 15 mai 1996 complétant l'Article 68 du Décret n81-039 du 2 février 1981 portant Code de déontologie des pharmaciens.

Décret N67-147 du 10 février 1967 instituant le Code de déontologie médicale.

Ministère de la Santé et de la prévention Médicale. Direction de la Pharmacie et des Laboratoires. 2006. Politique pharmaceutique nationale.

Ministère de la Santé et de l'action Sociale. Politiques, Normes et Protocoles en SR. Partie 2.
Ministère de la Santé et de l'action Sociale. Circulaire 008938 MSAS/ DGS/CSC du 11 août 2014 autorisant l'offre initiale de contraceptifs injectables sous forme intramusculaire (Depo Provera) et souscutanée (Sayana Press) au niveau communautaire.

Loi $n^{\circ}$ 54-418 du 15 avril 1954 étendant aux territoires d'Outre-Mer, au Togo et au Cameroun certaines dispositions du Code de la Santé Publique relatives à l'exercice de la pharmacie.

Loi 2005-18 du 5 août 2005 relative à la Santé de la Reproduction.

Protocole à la Charte africaine des droits de l'Homme et des peuples relatif aux droits des femmes en Afrique du 11 juillet 2003 (Protocole de Maputo).

Wickstrom, Jane, Abdoulaye Diagne, et Alyson Smith. 2006. Repositioning Family Planning-Senegal Case Study: Promising Beginnings, Uneven Progress. New York, NY, USA: EngenderHealth et The ACQUIRE Project.
Le Projet Evidence est rendu possible grâce au généreux support du Peuple Américain à travers l'Agence des Etats Unis pour le Développement International (USAID) sous les termes de l'accord de coopération no. AID-OAA-A-13-00087. Le contenu de ce document est la seule responsabilité du Projet Evidence et ne reflète pas nécessairement les points de vue de I'USAID ou du Gouvernement des Etats Unis.
Le Projet Evidence utilise la science de la mise en œuvre -la production, traduction, et utilisation stratégique d'évidences pour renforcer et mettre à l'échelle des programmes de planification familiale et de santé de la reproduction afin de réduire les grossesses non désirées partout dans le monde. Le Projet Evidence est dirigé par le Population Council en partenariat avec Population Reference Bureau.

\section{The Evidence Project}

Population Council

Sacré Cœur Pyrotechnie

Appartement 2ème Etage à Droite

Dakar

Sénégal

21027 Dakar Ponty

tél: +221.33.859.5300

evidenceproject@popcouncil.org
Citation recommandée: Mbow, Fatou Bintou, Ndeye Dome Fall, Nafissatou Diop, and Sara Chace Dwyer. 2018. "Exploration du rôle potentiel des pharmacies privées dans l'offre de services de planification familiale au Sénégal : Revue documentaire sur les aspects juridiques," Technical Brief. Dakar, Senegal: Population Council, Le projet évidence.

(C) 2018 Le Population Council, Inc.

Pour plus d'information, merci de contacter Fatou Bintou Mbow à fmbow@popcouncil.org 